\title{
combustión conjunta de fuel-oil y carbón *
}

\author{
ALFREDO SERRATOSA NADAL \\ de la Compañia Vaienciana de Cementos Portiana
}

Lo que se expone en el presente trabajo se refiere exclusivamente a la corta expcrien. cia que hemos tenido en nuestra fábrica de Buńol, con la combustión conjunta de carbón $y$ fuei-oil.

Ante todo debo indicar que la solución del empleo de mezcia de fuel-oil y carbón no fué una cosa deseada, sino más bien soportada o admitida sin gran entusiasmo. $\mathrm{Me}$ explicaré. En el año 1953 trabajábamos nosotros normalmente, como todos ustedes, con carbón, o mejor dicho, mezclas de car. bón y crudo, ya que el porcentaje de cenizas subía a cifras tan importantes, que se podía decir que en las cuencas carboneras teniamos una cantera auxiliar que nos daba muy cerca del 10 por 100 de materia prima, pero excesivamente cara.

Aprendimos a fabricar un cemento Portland con estos carbones, pero nuestra ciencia se vino abajo cuando intentamos lo mismo con la fabricación de cemento blanco, cuyas características especiales exigen unas primeras materias muy cuidadas y vigiladas en todos sus componentes, y muy especialmente en el contenido de hierro. La aportación de las cenizas deshacía todas las previsiones de los cálculos de la harina cruda, no sólo por el porcentaje de cenizas variable,

(-) Ponencia presentadia en los II Coloquios de Directores y Técnicos de Fábricas de Cemento. l. T. C. C.. nuarzo, 1957. aunque siempre elevado, sino que, por recibir los carbones de diferentes minas, la composición de esas cenizas cambiaba también con excesiva frecuencia. La forma especial de orǵanizar los suministros de carbón hacía inevitable estos graves inconvenientes.

Tuvimos que llegar, bien a pesar nuestro, a reducir la fabricación de cemento blanco, por encontrarnos impotentes ante ese problema.

Fué por entonces cuando se nos apuntó por la Superioridad, como posible solución, el que en las fábricas alejadas de las cuencas mineras se empleara el fuel-oil, máxime teniendo en cuenta que la destileria de Escombreras estaba trabajando y tenían un grave problema de almacenamiento de ese producto de bajo valor.

Hubo fuertes presiones para que nos decidiéramos por emplear ese combustible, ya que $l_{a}$ disponibilidad de hullas del Norte era insuficiente por problemas de extracción y de transporte, y si no instalábamos los quemadores podría ocurrir que llegáramos a tener que parar toda la fabricación por fajta de combustible.

Francamente he de confesar que, en aquellas circunstancias, el tener que emplear combustibles de importación, aunque fueran subproductos de una importante industria de destilación, no nos producia ninguna satisfacción. Si los combustibles españoles y clá- 
sicos en nuestra inclustria amenazaban cort faltarnos, parecía natural que los importados fueran todavía más inseguros.

Con ello quiero indicar con qué poco calor $y^{*}$ confianza tomamos $l_{\mathfrak{a}}$ sugerencia, si bien veíamos en el empleo de ese combustible, prácticamente sin cenizas, la úvica posibilidad de volver a fabricar un cemento blanco de las características que nosotros deseábamos y que ya habíamos conseguido en cuantas ocasiones dispusimos de combustibles apropiados.

Fuúmos, pues, casi empujatos al empleo del fuel-oil, pero ante este temor intimo que teníamos de que alguna vez nos quedáramos sin ese combustible, pensamos en instalar unos mecheros que, en caso de necesidad, y sin grandes maniobras ni paradas, pudiéramos hacer funcionar con carbón, como hasta entonces veniamos haciendo.

Consultamos a variás casas, y nos decidimos por una que nos daba garantía de perfecto funcionamiento según nuestros deseos.

Pero, a pesar de las garantías y seriedad de la casa constructora, teníamos nuestras dudas sobre la forma en que se comportarían dos combustibles de características $\tan$ distintas cono el fuel-oil y el carbón mezclados, sobre todo si llegábamos a emplear antracitas con sólo 10 por 100 de volátiles.

Parece que cada combustible debería arder en una zona distinta, y que, por tanto, no se conseguiría una zona definida de calcinación donde se tuviera la temperatura conveniente.

Por elio empezamos con fuel-oil solo, pero inmediatamente iniciamos la mezcla con carbón, debido a la alta temperatura que se tenía en la salida de clínker, con dificaltades en la boquilia de inyección y en la misma enfriadora Fuller, a la que llegaba el clínker todavía con gran cantidad de fase líquida, formando como una gran estalagmita en el punto de caída.
La adición de carbón se hizo primeramente con las mezclas normales con que trabajábamos, de grasos secos, y, a medida que nuestra confianza en el sistema iba creciendo, fuimos bajando la cantidad de graso, sin encontrar ninguna dificultad cuando liegamos a eliminarlo por completo.

Con la mezcla de fuel-oil y carbón, la zona de calcinación se alejó lo suficrente para eliminar los inconvenientes que se presentaron al quemar fuel-oil solo.

Fusé, pues, todo sencillo, como lo es también el fundamento de este sistema de combustión, que, en esencia, se reduce a pulverizar el fuel-oil en la corriente de aire primario cargada de polvo de carbón, en la proporción conveniente.

La instalación de dosificación e inyección de carbón no sufre ninguna variación, salvo el ajuste de la velocidad de los dosificadores, para una alimentación menor.

En el tubo de inyección del carbón se introduce un tubo de acero, que lleva el fuel a presión hasta la tobera de pulverización, en el extremo del tubo de inyección, y exactamente en el centro.

El fuel-oil pulverizado queda envuelto por la corriente de aire primario que lo inyecta dentro del horno.

El sistema de pulverización está fundado en la dispersión ocasionada en una lámina de fuel que va adelgazándose rápidamente.

Ei fuel-oil, que por calentamiento se lleva a una viscosidad de $2,5^{\circ}$ Engler, se inyecta a través de dos tanuras opuestas de $10 \times 2$ milimetros, tangencialmente, en una cámara cilindrica de unos $12 \mathrm{nim}$ de diámetro. La cantidad de fuel-oil que entra en esa cámara es del orden de $0,55 \mathrm{~kg} / \mathrm{seg}$, correspondiente a unos $15 \mathrm{~m} / \mathrm{seg}$. Como la cámara tiene tmos $12 \mathrm{~mm}$ de diámetro, est́a velocidad tangencial de enirada da al fuel-oil tuna velocidad de rotación del orden de las $\mathbf{3 0 . 0 0 0}$ r. p. m., aunque en realidad, por la viscosi- 
dad y roce con las paredes, quede bastante reducida.

El fuel a esa velocidad de rotación se inyecta al través de un crificio de mos $4 \mathrm{~mm}$ de diámetro: y por la composición de las fuerzas centrifugas y de inyección, ambas a $90^{\circ}$, se produce a la salida un cono formado por una lámina fina de fuel, que a poca distancia pierde su cohesión, transfcrmándose en una niebla constituída por pequeñísimas gotas. Es un sistema muy parccido at de las máquinas agrícolas de pulverización.

La presión en la cámara se puede hacer variar actuando sobre la válvula de descarga de la misma. Con ello se consigue variar el gasto a través del orificio de inyección, y se varía también, como es natural, el ángulo àel cono de salida.

Por ensayos realizados por la Casa constructora, con presiones de trabajo, antes del inyector, de $25 \mathrm{~kg} / \mathrm{cm}^{2}$, se obtiene una regulación de consumo, manteniendo una ex celente pulverización entre 1.650 y $100 \mathrm{~kg}$ por hora con variaciones de la presión de descarga comprendidas entre 19 y $2 \mathrm{~kg} / \mathrm{cm}^{2}$. En esas condiciones, el ángulo đel cono oscila entre $50^{\circ}$ para la presión mayor y $100^{\circ}$ para la menor.

Fácilmente se comprenden las posibilidades de este sistema de combustión.

La potencia calorífica del combustible pue. de pasar por toda la gama, desde el fuel solo con unas 9.800 calorías, hasta el carbón solo, cor 6.000 . Las cenizas pueden variar, paralelamente, de 0 a 20 ó 30 por 100. Los volátiles, de 100 a 10 por 100 , si se emplean antracitas. Estas variaciones pueden conseguirse inmediatamente, a voluntad, actuando sobre la alimentación de fuel-oil y carbón.

Normalmente nosotros hemos trabajado con proporciones de una parte de fuel por una a dos partes de carbón. Con ello tene- mos combustible de 7.900 a 7.200 calorías, $y$ de 10 a 14 por 100 de cenizas.

Podría parecer que el régimen de aire de combustión, y consiguientemente el de inyección, tuvieran que variar dentro de límites extensos ; pero, en la práctica no es así.

La mayor cantidad de aire que necesita 1 kilogramo de fuel-oil, de an orden aproximado a $14 \mathrm{~kg}$, frente a 11 del carbón, queda compensada, en parte, por la mayor potencia calorífica de aquél; y en las mezclas empleadas en la práctica por nosotros, la cantidad de aire por unidad de calor es sólo un 6 por 100 mayor, cuando se emplea una mezcla mayor de carbón.

La inyección de la mezcla de fuel-oil y carbón dentro del horno se hace por el aire primario que nosotros llevamos a unos 40 $\mathrm{m} / \mathrm{seg}, y$ en la proporción del 20 por 100 sobre el atire total necesario, obteniendo, con ello, una llama larga brillante, no excesivamente caliente, que no puede castigar con exceso el revestimiento refractario.

Por dicho motivo, la duración de dicho revestimiento es prácticamente la misma que cuando se empleaba carbón.

En cuanto a calidad de clínker, lo mismo que en lo referente a producción, es indudable que hay mejora, sobre todo cuando se compara con la obtenida con carbones de baja calidad, con los que han trabajado bastantes fábricas.

Sabemos de algunas que han recibido carbones de 40 por 100 de cenizas y que, aun conteniendo un buen porcentaje de agua, no creemos que hubieran sido lavados previamente.

La difetencia entre ese carbón y el combustible que se puede conseguir con mezclas convenientes es tan grande que, indudabiemente, la marcha del horno ha de mejorar sustancialmente. Las temperaturas y características de la llama se manejan fácilmente, actuando sobre los tres elementos: fuel-oil, 
carbón y aire, y, al mismo tiempo, las cenizas tienen una infiuencia mucho más reducida, ya que puede considerarse, sin gran error, que se reducen a una cuarta o quinta parte de las del carbón anteriormente indicado como límite de mala calidad.

La instalación del sistema de atomización no es demasiado complicada, si bien no hay que pensar que sea una cosa sin importancia.

E1 problema principal se reduce a pulverizar el fuel-oil. Este combustible, por el precio a que se consiguió, mediante gestiones en las que jugó un papel importante el sefíor Fonrodona, es, o puede ser un producto en la úitima escala de la destilación de crudos. Como tal, su calidad es muy variable; se recibe lo que a uno le envían, ya que el calificativo de fuel-oil núm. $\mathbf{2}$, es, según creo, lo peor, $y$ con darnos lo peor, se ha cumplido.

Sin embargo, he de confesar que, hasta ahora, la clasificación ba estado más en el papel que en la realidad. En la última fijación de precios de fuel-oil no se tiene en cuenta la calidad, sino el empleo, to que no deja de ser una posición anormal.

Como en todas las sittaciones ficticias $y$ anormales existe el peligro de volver a la realidad y normalidad, ¿Qué podría ocurrir entonces?

1. Que el fuel 2 desapareciese, con el consiguiente aumento de precio, lo que sería un desastre económico.

2. Que el combustible sea de muy baja calidad, con lo que sólo las instalaciones que estén proyectadas para ello puedan funcionar.

Lo anteriormente indicado ha de tenerse muy en cuenta para cualquier instalación que se proyecte. Las fábricas de cemento están conceptuadas como fábricas que pueden em. plear los peores combustibles para su desenvolvimiento. En algunas ocasiones he tenido que oir -por personas que se dicen cnteradas- que el contenido de cenizas de carbón era interesante, pues con ellas se aumentaba la producción, al quedar incorporadas at clínker.

Hay que esperar, si Dios no lo remedia, le toque el turno al fuel-oil, y sea lo peor de lo peor. En ese caso, el problema de atomización tendrá dificultades que habrá que resolver estando preparados para ello.

Para el trasvase y circulación, hay que calentar a temperatura conveniente, del orden de $80^{\circ}$ actualmente, y quizá más en el porvenir.

Si el calor específico del fuel-oil es de $0,38 \mathrm{kcal} / \mathrm{kg}$ y por término medio hay que elevar, en el peor de los casos, a $100^{\circ} \mathrm{C}$, se necesitarán 38 calorías por $\mathrm{kg}$ ó $38.000 \mathrm{kcal} /$ tonelada.

Como no hay que contar con un rendimiento superior al $\mathbf{5 0}$ por $\mathbf{1 0 0}$ en todo el sistema de calefacción, hay que pensar en, por lo menos, ttn gasto de $76.000 \mathrm{kcal} / \mathrm{Tm}$, o sea, empleando fuel-oil: $76.000 / 9.800=8 \mathrm{~kg}$ de fuel por tonelada consumida en el horno, por cada una de las operaciones de transvase que se hagan, y que pueden variar, según la disposición de cada fábrica, entre 1 y 3 y, por tanto, de 8 a $24 \mathrm{~kg}$ de fuel por tonelada de fuel consumido en el horno.

El sistema más corriente es el empleo de vapor a 8 ó 10 atmósferas, por lo que hay que prever una caldera de capacidad de 125 a $350 \mathrm{~kg}$ de vapor por hora y por tonelada de fuel-oil.

La caldera, como es natural, lleva sus problemas de depuración de agua y recuperación de condensaxtos, to que conviene tener presente para el proyecto completo de la instalación.

Para la atomización, según el sistema em. pleado en Buñol, hay que comprimir el fuel a unas 25 atmósferas y hacerlo pasar por la tobera de $4 \mathrm{~mm}$ de diámetro, con una viscosidad de 2,5 Engler, to que presupone 
una temperatt1ra entre 80 y $90^{\circ} \mathrm{C}$, con los fuels actuales.

Esta operación obliga a :

1. Filtrar el fuel,

2. Calentarlo.

3. Bombearlo.

La instalación consiste en :

a) Un circuito de alimentación, compuesto de:

1 depósito de alimentación de fuel de unas 10 toneladas de capacidad

1 manómetro a la salida de la bomba

1 filtro doble

1 bomba (doble)

1 circuito a presión de 25 a $30 \mathrm{~kg} / \mathrm{cm}^{2}$

1 calentador doble

1 contador

1 tuberia de distribución

1 válvula de alimentación del atomizador

1 atomizador

1 válvula de descarga del atomizador

1 válvula reguladora de presión

1 by-pass para la puesta en marcha

b) Una tubería de descarga que recoge $\mathrm{e}$ fuel sobrante, a través de las válvulas reguladoras y de los atomizadores, y lo lleva a la aspiración de la bomba de inyección.

Antes de ésta se tiene un contador que registra el fuel de retorno, con lo que, por diferencia, se puede deducir el fuel consumido.

Aparte de 1o anteriormente indicado hay que contar con la correspondiente instalación de depósitos de almacenaje, cuya capacidad depende de varios factores y, principalmente, de la regularidad de los suministros de la CAMPSA.

Nosotros aconsejariamos, como mínimo, el suministro de un mes.

Por lo anteriormente expuesto, se deduce que la instalación de un quemador de fuel tiene bastante más importancia de io que a primera vista puede parecer.
Pasemos ahora al asunto de su mezcla con carbón pulverizado.

Empezaré por el suministro de carbón.

No voy a describir lo que ello significa, sobre todo si, como en la zona mediterránea, se tiene que contar con el empleo de todas las clases de carbones, ya que no se tiene ninguna cuenca específica.

Desde ht1las de Asturias y antracitas de León, hasta carbones de Puertollano y de otras cuencas, se puede decir que todo es posible que pase por nuestras fábricas.

Las características son bien distintas, no solamente en cuanto a composición y potencia calorífica, sino en cuanto a tamaño, tendencia a aglomerarse y pegarse, tipo de vagones empleado en el transporte, irregularidad de abastecimiento, etc.

Todo ello obliga a complicar la instatación de la sección de carbones, desde los medios de descarga y almacenaje, hasta los de secado y molienda.

Se comprende, pues, que en las condiciones en que se desenvuelve el suministro de combustibles sólidos y liquidos, la solución del empleo mixto de los mismos es técnicamente excelente, pero desde el punto de vista económico, ya no lo es tanto. Los gastos de primera instalación son muy elevados, ya que hay que calcular dos instalaciones completas para una elasticidad de servicio entre límites muy extensos, pues cada instalación ha de poder ser suficiente para atender ella sola todas las exigencias de la fabricación.

Ello se complica más si son varios los hornos que hay que alimentar.

Los rendimientos, pues, de las instalaciones, no pueden ser los más apropiados y, por consiguiente, no se puede pretender, en general, tina buena productividad de la mano de obra ni de la maquinaria, en esas secciones específicas, ya que se tiene una capacidad de prodtucción que normalmente sólo se aprovecha en un $\breve{00}$ por 100 cuando se trata 
de an solo homo, disminuyendo en progresión geométrica cuando aumenta el número de hornos.

Sin que quiera exagerar la nota pesinista, a la ventaja de la gran clasticidad de posíbilidades de trabajo con cualqquier mezcla de combustibles, se opone el peligro de que consideren la fábrica aś utilluda como la fábrica comodin, que puede superar todas las dificultades y que, por lo mismo, a ella le van todos los problemas.

Hemos estado, con frecuencia, con la preocupación de quedarnos sin fuel; pues, como podemos ir con carbón... Otras veces, al contrario, sin carbón : porque, como podemos ir con fuel... Y hasta han llegado momentos en que han coincidido las dos tendencias y el peligro era quedarnos sin una cosa $y$ sin otra.

Como es natural, la elasticidad de este sistema de combustión no llega a tanto.

Todo cambio lieva sus dificultades, ya que, desde la adquisición del combustible hasta su empleo, hay una serie escalonada de probIemas cuya solución no se puede improvisar. Los planes de trabajo se han de pre. parar con tiempo y no es posible valriarlos continuamente.

No hay que olvidar, pues, este aspecto de las fábricas cuyas instalaciones están concebidas de forma que parezca que sirven fácilmente para todo.

Pasemos a considerar el muy importante factor económico.

En cada caso particular hay que mirar que la caloría sea económica y segura para tener una continutidad en la fabricación.

Cada fábrica será un problema distinto.

Quien tenga un carbón barato y próximo, no debe pensar en el empleo de fuel. Quien sólo tenga posibilidades de un mal carbón, con un transporte costoso de cenizas y agua, con inseguridades de suministro y con la circunstancia de proximidad a depósitos de
CAMPSA, puede pensar en la solución de empleo de fuel, solo o mezclado con carbón. De todas formas hay que calcular que el precio de la calotía del fuel es, en general, más elevado que el de la de carbón.

Alora bien, en mezclas de ambos combustibles pueden emplearse carbones de pocos volátiles, que solos no podrían consumirse, to que en algunos casos puede ser ventajoso.

En resumen, que cada caso particular debe estudiarse detenidamente, teniendo en cuenta todas las circunstancias que concurren en el suministro de combustibles. La solución mejor, muchas veces, no coincidirá con el deseo de uno.

No quiero terminar sin, por lo menos, señalar el problema de la formación de anillos.

Entre los múltiples motivos de formación de los mismos está el de las cenizas de los carbones.

Antes de1 empleo del fuel teníamos formaciones de anilios, si bien lo encontrábamos natural, dado que empleábamos carbones con más del 30 por 100 de cenizas.

Dichos anilios presentaban una estructura hojosa, formada por capas de material muy distinto. Unas capas estaban completamente fundidas y aprisionaban otras, formadas como de granos de clínker o bien de material que se pulverizaba.

La explicación de esta estructura sólo cabe suponiendo que se forman avenidas de material mtiy fusible, que sirve de base a una pegadtura que aprisiona material más tefractario.

E1 análisis de las diferentes capas dió unas acusadas diferencias en su composición.

Las capas que servian de base para la pe: gadura tenían una gran proporción de sílice, alúmina y hierro, con una disminución muy pronunciada de cal, manteniéndose normal la magnesia, 
Los granos aprisionados eran prácticamente de composición igual al clínker. La diferencia que puede existir hay que atribuitla más bien a la forma de tomar la muestra.

Creimos firmemente que, con la clminación de gran parte de las cenizas del carbón, al emplear fuel, no se nos produciría este fenómeno. La realidad, sin embargo, nos ha convencido de que nuestras previsiones no eran ciertas.

Los anillos se han vuelto a producir, autque no con la frecuencia anterior, pero la composición de los mismos ha sido diferente.

Se ha observado una disminución de cal y un aumento de $\mathrm{SiO}_{2}$ y $\mathrm{Al}_{2} \mathrm{O}_{3}$ y, sobre todo, un aumento o aparición exagerada de $\mathrm{SO}_{3}$, que llega a 9 por 100 en el anillo.

Aprovechando una parada del horno se hizo una inspección del mismo, encontranđo, detrás de la zona de calcinación y de la parte en la que normalmente se forman los anillos, una zona tevestida de material de aspecto pulverulento.

La composición de la costra daba un análisis en el que se notaba una gran disminución de la cal (con 50,60 por 100) y un contenido elevadísimo de $\mathrm{SO}_{3}$ (de 11,50 por 100).

En otra parada del horno, y en la cámara de la parrilla de recuperación, se observaron unos depósitos de material que, sin pérdida al fuego apreciable, tenian una composición verdaderamente notable, con:

$$
\begin{aligned}
& \mathrm{SiO}_{3} \ldots \ldots \ldots=28,60 \\
& \mathrm{Al}_{2} \mathrm{O}_{3} \ldots \ldots \ldots=14,00 \\
& \mathrm{Fe}_{2} \mathrm{O}_{3} \ldots \ldots \ldots=5,10 \\
& \mathrm{CaO} \ldots \ldots \ldots=29,40 \\
& \mathrm{MgO} \ldots \ldots \ldots=1,80 \\
& \mathrm{SO}_{3} \ldots \ldots \ldots=13,50
\end{aligned}
$$

en donde se observa un módulo silícico de 1,5 y un módulo hidráulico de 0,63 .

Dicho material, por su composición, ha de ser muy fusible y, a nuestro modo de ver, puede ser el motivo de la formación de anillos.

Por la forma especial de trabajar el horno Lepol es muy fácil la formación de incrustaciones y depósitos del polvo en suspensión en los gases, en los puntos más frios y en donde la velocidad y dirección de los gases tienen variaciones.

Cuando el depósito de estos materialcs tiene suficiente peso, debe haber desprendimientos masivos sobre la parrilla que alimenta el horno, y, al llegar a la zona caliente, ese material se funde, sirviendo de base a la formación del anillo.

El proceso se repite a intervalos, que son más frecuentes a medida que los depósitos han sido mayores en los puntos en los que las condiciones locales favorecen la acumulación de ese polvo de composición anormal.

La exudación de la fase líquida, junto con la aportación de nuevas cantidades de material fusible, acelera el proceso de formación de los anillos.

Esta particular hipótesis de la formación de anillos parece confirmarse por el hecho de que los mismos no se inician hasta después de unos días de marcha del horno, o sea, hasta que ha habido tiempo de formarse los depósitos de material fusible en las cámaras de la parrilla o en las paredes de la parte más fría del horno.

Por otra parte, estas acumulaciones de pol. ro quedan muy favorecidas por los cambios bruscos de dirección de los gases y por la menor velocidad de los mismos.

Esl horno Lepol, por su menor consumo de combustible y por su especial conducción de gases, se presta mucho a este proceso de precipitación.

Con lo anteriormente indicado, queremos poner de manifiesto que, aunque con el em. pleo de fuel se reducen las posibilidades de formación de anillos, no hay que creer que quedan completamente eliminadạs. 
Insistimos, sin embargo, en que es muy expuesto generalizar sobre resultados obtenidos en unas u otras fábricas y que, aunque las experiencias de las diferentes fábricas deben ayudat a resolver los problemas que se pueden presentar, no hay que pensar en que la solución de una sea lá mejor solución para otra.

Ahora bien, este cambio de ideas y estos coloquios entre los interesados en el mismo asunto, 10 creemos francamente constructivo y de gran interés, en tanto no se salgan de lo que esta palabra significa: una conversación cordial, sincera $y$ sin reservas, en la que cada tho exponga sus problemas y las soluciones que, con más o menos fortuna, haya encontrado para los mismos.

Esta ha sido la intención perseguida en mi exposición, y ruego a los que han tenido la deferencia y paciencia de seguirme que colaboren con la segunda parte de la misma, a sea, en la discusión de todo lo dicho anteriormente con más o menos acierto, pero desde litego con toda sinceridad. 